Journal of Data Science 13(2015), 261-280

\title{
A COMPARATIVE STUDY BASED ON BAYES ESTIMATION UNDER DIFFERENT CENSORING CRITERION
}

\author{
Gyan Prakash. ${ }^{1 *}$ \\ ${ }^{1}$ Department of Community Medicine, S. N. Medical College, Agra, U. P., India
}

Abstract:The censoring arises when exact lifetimes are only partially known, and it is useful in life testing experiments for time and cost restrictions. Especially, when some sample values at either or both extremes might have been adulterated. In present article, the Bayes estimation for unknown parameter of Gompertz distribution has been addressed based on three different censoring criterions. The performances of the procedures are illustrated by a simulation technique.

\section{Introduction}

Gompertz (1825) first introduced the underlying model, and its distribution and probability density function are defined as

$$
F(x ; \theta)=1-\exp \left\{-\theta e^{x}+\theta\right\} ; \theta>0, x>0
$$

and

$$
f(x ; \theta)=\theta e^{x} \exp \left\{-\theta e^{x}+\theta\right\} ; \theta>0, x>0,
$$

where the parameter $\theta$ is known as scale parameter.

Gompertz probability distribution has many useful applications in areas of technology, medical, biological, and natural sciences (especially in failure and survival analysis). This distribution also widely used in the model of human mortality and fit in the actuarial tables.

Based on progressive first-failure censoring plan Soliman et al. (2012) studied the Bayes and frequentist estimators for two-parameter Gompertz distribution. Ismail (2010) discussed the Bayes estimation for unknown parameters of Gompertz distribution and acceleration factor under partially accelerated life tests under Type-I censoring. They applied Bayesian approach for estimation problem in case of step stress partially accelerated life tests for two stress levels and under Type-I censoring.

$\mathrm{Wu}$, et al. (2003) discussed about point and interval estimations for Gompertz distribution under Progressive Type-II censoring. Jaheen (2003) discussed about the Bayesian analysis of record statistic from Gompertz model. The maximum likelihood (ML) estimation for mixtures of two Gompertz distributions when censoring occurs has derived by Gordon (1990). Based on human mortality model, some parameter estimation of Gompertz population was discussed by Chen (1997). Anands et al. (1996) derived some adaptive Bayes estimators for the parameters of

${ }^{*}$ Corresponding author. 
Gompertz survival model. They present Bayes estimators for the parameters in terms of single numerical integrations.

Bayesian analysts have pointed out that there is no clear-cut way from which one can conclude that one prior is better than the other. It is more frequently the case that, a prior is selected to restrict attention to a given natural family of priors, and one is chosen from that family, which seems to match best with one's personal beliefs. However, if one has adequate information about the parameter, one should use informative prior; otherwise it is preferable to use noninformative prior. The Gamma distribution is considered here as a conjugate family of prior for unknown parameter $\theta$, having probability density function

$$
\pi_{1}(\theta)=\frac{c^{b}}{\Gamma b} \theta^{b-1} e^{-c \theta} ; b, c>0, \theta \geq 0 .
$$

The Jeffrey's prior is the example of non-informative prior which materializes the use of Bayesian estimation methods when no prior information is available. In present case, the second prior is considered as the non-informative (Jeffrey's) prior and is defined as

$$
\pi_{2}(\theta) \propto \theta^{-1} ; \theta>0 .
$$

The selection of loss function may be crucial in Bayesian analysis also. If most commonly used loss function, squared error loss function (SELF) is taken as a measure of inaccuracy then the resulting risk is often too sensitive to assumptions about the behavior of tail of probability distribution. Also the use of SELF in Bayesian estimation may not be appropriate in case when positive and negative errors have different consequences. In addition, in some estimation problems overestimation is more serious than underestimation, or vice-versa. A useful and flexible class of asymmetric loss function (LINEX loss function (LLF)) is defined for such cases as

$$
L\left(\partial^{*}\right)=e^{a \partial^{*}}-a \partial^{*}-1 ; a \neq 0, \partial^{*}=(\hat{\theta}-\theta) .
$$

Here, $\hat{\theta}$ be any estimate corresponding to unknown parameter $\theta$ and ' $a$ ' be the shape parameter of LLF. Negative (positive) value of ' $a$ ', gives more weight to overestimation (underestimation) and its magnitude reflect the degree of asymmetry. It is seen that, for $a=1$, LLF is quite asymmetric with overestimation being more costly than underestimation. For small values of $|a|$, LLF is almost symmetric and is not far from SELF.

In many life testing experiments, the experimenter may not be observed the lifetimes of all inspected units in life test. This may be because of time limitation and/or cost or material resources on data collection. Also, the trimmed samples are widely utilized when some sample values at either or both extremes adulterated.

The aim of present paper is to present a comparative study based on Bayes estimation for unknown parameter under different censoring plans. For this we consider here three different censoring schemes wiz, Item-Failure (Type-II), Doubly Type-II and Progressive Type-II right censoring. Bayes estimation has been addressed for Gompertz model under LINEX loss function. 
The performances of the procedures are illustrating by a simulation technique. A real data (King et al. (1979)) example is also provided to illustrate the proposed methods. A brief conclusion is presented at the end.

\section{Bayes Estimation of Unknown Parameter under Informative Prior}

\subsection{Item Failure Censoring}

In life testing, fatigue failures and other kinds of destructive test situations, the observations usually occurred in ordered manner such a way that weakest items failed first and then second one and so on. Let us suppose that $n$ items are put to test under the model (1.1) without replacement and test terminates as soon as first $r^{\text {th }}(r \leq n)$ item fails. This censoring scheme is known as Item-Failure censoring scheme.

Now, $x_{(1)}, x_{(2)}, \ldots, x_{(n)}$ be $n$ ordered items assumed from model (1.1). If $\underline{x}=$ $\left(x_{(1)}, x_{(2)}, \ldots, x_{(r)}\right)$ be first $r$ components of the observed failure items, then joint probability density function for these order statistics is defined as

$$
\begin{aligned}
f_{\left(X_{(1)}, X_{(2)}, \ldots, X_{(r)}\right)}(\underline{x} \mid \theta) & =\prod_{i=1}^{r} f\left(x_{(i)} ; \theta\right) \cdot\left(1-F\left(x_{(r)} ; \theta\right)\right)^{n-r} \\
& =\prod_{i=1}^{r}\left(\theta e^{x_{(i)}} \exp \left(-\theta\left(e^{\left(x_{(i)}\right)}-1\right)\right)\right) \cdot\left(\exp \left(-\theta\left(e^{\left(x_{(r)}\right)}-1\right)\right)\right)^{n-r} \\
\Rightarrow f_{\left(X_{(1)}, X_{(2)}, \ldots, X_{(r)}\right)}(\underline{x} \mid \theta) & =\theta^{r}\left(A_{I}^{*}(\underline{x})\right) \exp \left(-\theta T_{I}^{*}(\underline{x})\right)
\end{aligned}
$$

where $A_{I}^{*}(\underline{x})=\exp \left(\sum_{i=1}^{r} x_{(i)}\right)$ and $T_{I}^{*}(\underline{x})=\sum_{i=1}^{r}\left(e^{x_{(i)}}-1\right)+(n-r)\left(e^{x_{(r)}}-1\right)$.

Based on Bayes theorem, the posterior density is now defined as

$$
\pi_{I C}^{*}(\theta \mid \underline{x})=\frac{f_{\left(X_{(1)}, X_{(2)}, \ldots, X_{(r)}\right)}(\underline{x} \mid \theta) \cdot \pi_{1}(\theta)}{\int_{\theta} f_{\left(X_{(1)}, X_{(2)}, \ldots, X_{(r)}\right)}(\underline{x} \mid \theta) \cdot \pi_{1}(\theta) d \theta} .
$$

Using (2.1) and (1.2) in (2.2), the posterior density is obtain as 


$$
\begin{aligned}
& \pi_{I C}^{*}(\theta \mid \underline{x})=\frac{\theta^{r}\left(A_{I}^{*}(\underline{x})\right) e^{-\theta T_{I}^{*}(\underline{x})} \cdot \frac{c^{b}}{\Gamma b} \theta^{b-1} e^{-c \theta}}{\int_{\theta} \theta^{r}\left(A_{I}^{*}(\underline{x})\right) e^{-\theta T_{I}^{*}(\underline{x})} \cdot \frac{c^{b}}{\Gamma b} \theta^{b-1} e^{-c \theta} d \theta} \\
& \Rightarrow \pi_{I C}^{*}(\theta \mid \underline{x})=\frac{\left(T_{I}^{*}(\underline{x})+c\right)^{r+b}}{\Gamma(r+b)} \theta^{r+b-1} e^{-\theta\left(T_{I}^{*}(\underline{x})+c\right)} .
\end{aligned}
$$

The Bayes estimator $\hat{\theta}_{I C}$ for unknown parameter $\theta$ under LLF is obtain by simplifying following equality

$$
\begin{aligned}
& \hat{\theta}_{I C}=-\frac{1}{a} \log E\left(e^{-a \theta}\right) \\
& \Rightarrow \hat{\theta}_{I C}=\left(\frac{r+b}{a}\right) \log \left(1+\frac{a}{T_{I}^{*}(\underline{x})+c}\right) .
\end{aligned}
$$

\subsection{Doubly Type-II Censoring}

The plan of experiments with aim of reducing total duration or number of failures leads naturally to Type-I \& Type-II censoring scheme. The main disadvantage of these censoring schemes is that they do not allow removal of units at points other than termination point of an experiment.

In addition, sometime, some data may not be observed, a known number of observation in an ordered sample are missing at both ends in failure censored experiments, observations are smallest and largest, are random then data collected will be. In such case doubly censoring criterion is useful.

Consider a life-test experiment in which ${ }^{n}$ identical units are placed on a test from the (1.1). The first $r$ units may be left censored due to negligence or problems at beginning of the experiment, and experiment terminates as soon as the $s^{\text {th }}$ unit failed. Then the data $x_{(r)}, x_{(r+1)}, \ldots, x_{(s)}$ constitute a Doubly Type-II censored sample in which $(r-1)$ smallest observations and $(n-s)$ largest observations have been censored. If $\underline{x}=\left(x_{(r)}, x_{(r+1)}, \ldots, x_{(s)}\right)$ be the observed failure items, then the joint probability density function for these order statistics is defined as

$$
f_{\left(X_{(r)}, X_{(r+1)}, \ldots, X_{(s)}\right)}(\underline{x} \mid \theta)=\frac{n !}{(r-1) !(n-s) !}\left(F\left(x_{(r)} ; \theta\right)\right)^{r-1}\left(1-F\left(x_{(s)} ; \theta\right)\right)^{n-s} \prod_{i=r}^{s} f\left(x_{(i)} ; \theta\right) ;(2 . s
$$

where $f(\cdot)$ and $F(\cdot)$ be the corresponding probability density and distribution function respectively. Simplifying (2.5), we get 


$$
\Rightarrow f_{\left(X_{(r)}, X_{(r+1)}, \ldots X_{(s)}\right)}(\underline{x} \mid \theta) \propto \sum_{k=0}^{r-1}(-1)^{k}\left(\begin{array}{c}
r-1 \\
k
\end{array}\right) \theta^{s-r+1} \exp \left(-\theta T_{D}^{*}(\underline{x})\right)
$$

where $T_{D}^{*}(\underline{x})=\sum_{i=r}^{s}\left(\mathrm{e}^{x_{(i)}}-1\right)+k\left(\mathrm{e}^{x_{(r)}}-1\right)+(n-s)\left(\mathrm{e}^{x_{(s)}}-1\right)$.

Applying Bayes theorem, the posterior density is obtained as

$$
\begin{aligned}
& \pi_{D C}^{*}(\theta \mid \underline{x})=\frac{\sum_{k=0}^{r-1}(-1)^{k}\left(\begin{array}{c}
r-1 \\
k
\end{array}\right) \theta^{s-r+1} \exp \left(-\theta T_{D}^{*}(\underline{x})\right) \cdot \theta^{b-1} e^{-c \theta}}{\int_{\theta}^{r-1}(-1)^{k}\left(\begin{array}{c}
r-1 \\
k
\end{array}\right) \theta^{s-r+1} \exp \left(-\theta T_{D}^{*}(\underline{x})\right) \cdot \theta^{b-1} e^{-c \theta} d \theta} \\
& \Rightarrow \pi_{D C}^{*}(\theta \mid \underline{x})=\eta \sum_{k=0}^{r-1}(-1)^{k}\left(\begin{array}{c}
r-1 \\
k
\end{array}\right) \theta^{s-r+b} \exp \left(-\theta\left(T_{D}^{*}(\underline{x})+c\right)\right) ;
\end{aligned}
$$

where $\eta=\left(\sum_{k=0}^{r-1}(-1)^{k}\left(\begin{array}{c}r-1 \\ k\end{array}\right) \frac{\Gamma(s-r+b+1)}{\left(T_{D}^{*}(\underline{x})+c\right)^{(s-r+b+1)}}\right)^{-1}$.

The Bayes estimator $\hat{\theta}_{D C}$ for unknown parameter $\theta$ under LLF is given as

$$
\hat{\theta}_{D C}=-\frac{1}{a} \log \left(\eta^{*} \sum_{k=0}^{r-1}(-1)^{k}\left(\begin{array}{c}
r-1 \\
k
\end{array}\right)\left(T_{D}^{*}(\underline{x})+c+a\right)^{-(s-r+b+1)}\right)
$$

where $\eta^{*}=\left(\sum_{k=0}^{r-1}(-1)^{k}\left(\begin{array}{c}r-1 \\ k\end{array}\right)\left(T_{D}^{*}(\underline{x})+c\right)^{-(s-r+b+1)}\right)^{-1}$.

\subsection{Progressive Type-II Censoring}

The progressive censoring appears to be a great importance in planned duration experiments in reliability studies. In many industrial experiments involving lifetimes of machines or units, experiments have to be terminated early and the number of failures must be limited for various reasons.

Let us suppose an experiment in which $n$ independent and identical units $x_{1}, x_{2}, \ldots, x_{n}$ are placed on a life test at the beginning time and first $r ;(1 \leq r \leq n)$ failure times are observed. At the time of each failure occurring prior to the termination point, one or more surviving units are removed from the test. The experiment is terminated at time of $r^{\text {th }}$ failure, and all remaining surviving units are removed from the test. 
Let $x_{(1)} \leq x_{(2)} \leq \ldots \leq x_{(r)}$ are the lifetimes of completely observed units to fail and $R_{1}, R_{2}, \ldots, R_{r} ;(r \leq n)$ are the numbers of units withdrawn at these failure times. Here, $R_{1}, R_{2}, \ldots, R_{r} ;(r \leq n)$ all are predefined integers follows the relation

$$
\sum_{j=1}^{r} R_{j}+r=n
$$

At first failure time ${ }^{x_{(1)}}$, withdraw $R_{1}$ items randomly from remaining $n-1$ surviving units. Immediately after the second observed failure time ${ }^{x_{(2)}}, R_{2}$ items are withdrawn from remaining $n-2-R_{1}$ surviving units at random, and so on. Experiments continue until at $r^{\text {th }}$ failure time $x_{(r)}$, remaining items $R_{r}=n-r-\sum_{j=1}^{r-1} R_{j}$ are withdrawn. Here, $\quad x_{1: r \cdot n}^{\left(R_{1}, R_{2}, \ldots R_{r}\right)}, x_{2: r: n}^{\left(R_{1}, R_{2}, \ldots R_{r}\right)}, \ldots$, $x_{r: r: n}^{\left(R_{1}, R_{2}, \ldots R_{r}\right)}$ and $\left(R_{1}, R_{2}, \ldots, R_{r}\right)$ be the $r$ ordered failure times and progressive censoring scheme respectively (Balakrishnan \& Aggarwala, 2000).

The resulting $r$ ordered values, which are obtained as a consequence of this type of censoring, are appropriately referred to as Progressively Type-II right censored order statistics. Progressively Type-II right censoring scheme reduces to conventional Type-II censoring scheme when

$$
R_{i}=0 \forall i=1,2, \ldots, r-1 \Rightarrow R_{r}=n-r
$$

and for

$$
R_{i}=0 \forall i=1,2, \ldots, r \Rightarrow n=r
$$

above censoring scheme reduces to complete sample case.

Based on Progressively Type-II censoring scheme the joint probability density function of order statistics $x_{1: r: n}^{\left(R_{1}, R_{2}, \ldots, R_{r}\right)}, x_{2: r: n}^{\left(R_{1}, R_{2}, \ldots, R_{r}\right)}, \ldots, x_{r: r: n}^{\left(R_{1}, R_{2}, \ldots R_{r}\right)}$ is defined as

$$
f_{\left(X_{(1: r: n)}, X_{(2: r: n)}, \ldots, X_{(r: r: n)}\right)}(\underline{x} \mid \theta)=C_{p} \prod_{i=1}^{r} f\left(x_{(i)} ; \theta\right)\left(1-F\left(x_{(i)} ; \theta\right)\right)^{R_{i}}
$$

The progressive normalizing constant $C_{p}$ is defined as $C_{p}=n\left(n-R_{1}-1\right)\left(n-R_{1}\right.$ $\left.-R_{2}-2\right) \ldots\left(n+1-\sum_{j=1}^{r-1} R_{j}-r\right)$. Here, $\underline{x} \equiv\left(x_{(1)}, x_{(2)}, \ldots, x_{(r)}\right)$ be the Progressive Type-II censored sample and $\left(R_{1}, R_{2}, \ldots, R_{r}\right)$ being progressive censoring scheme of the considered model. Simplifying (2.9) we have

$$
\Rightarrow f_{\left(X_{(1: r: n)}, X_{(2: r: n)}, \ldots, X_{(r: r: n)}\right)}(\underline{x} \mid \theta)=C_{p} A_{P}^{*}(\underline{x}) \theta^{r} \exp \left(-\theta T_{P}^{*}(\underline{x})\right) ;
$$


where $A_{p}^{*}(\underline{x})=\exp \left(\sum_{i=1}^{r} x_{(i)}\right)$ and $T_{p}^{*}(\underline{x})=\sum_{i=1}^{r}\left(1+R_{i}\right)\left(\mathrm{e}^{x_{(i)}}-1\right)$.

Applying Bayes theorem, the posterior density is obtained as

$$
\begin{aligned}
& \pi_{P C}^{*}(\theta \mid \underline{x})=\frac{C_{p} A_{P}^{*}(\underline{x}) \theta^{r} \exp \left(-\theta T_{P}^{*}(\underline{x})\right) \cdot \frac{c^{b}}{\Gamma b} \theta^{b-1} e^{-c \theta}}{\int_{\theta} C_{p} A_{P}^{*}(\underline{x}) \theta^{r} \exp \left(-\theta T_{P}^{*}(\underline{x})\right) \cdot \frac{c^{b}}{\Gamma b} \theta^{b-1} e^{-c \theta} d \theta} \\
& \Rightarrow \pi_{P C}^{*}(\theta \mid \underline{x})=\frac{\left(T_{P}^{*}(\underline{x})+c\right)^{r+b}}{\Gamma(r+b)} \theta^{r+b-1} e^{-\theta\left(T_{P}^{*}(\underline{x})+c\right)} .
\end{aligned}
$$

The Bayes estimator $\hat{\theta}_{P C}$ for the parameter $\theta$ under LLF is given as

$$
\Rightarrow \hat{\theta}_{P C}=\left(\frac{r+b}{a}\right) \log \left(1+\frac{a}{T_{P}^{*}(\underline{x})+c}\right) \text {. }
$$

\section{Bayes Estimation under Non-Informative Prior}

\subsection{Item Failure Censoring}

Using joint probability density function (2.1) and equation (2.2), the posterior density corresponding to the Jeffrey's prior (1.3) is obtained as

$$
\begin{aligned}
& \pi_{I C}^{* *}(\theta \mid \underline{x})=\frac{\theta^{r}\left(A_{I}^{*}(\underline{x})\right) e^{-\theta T_{I}^{*}(\underline{x})} \cdot \theta^{-1}}{\int_{\theta} \theta^{r}\left(A_{I}^{*}(\underline{x})\right) e^{-\theta T_{I}^{*}(\underline{x})} \cdot \theta^{-1} d \theta} \\
& \Rightarrow \pi_{I C}^{* *}(\theta \mid \underline{x})=\frac{\left(T_{I}^{*}(\underline{x})\right)^{r}}{\Gamma(r)} \theta^{r-1} e^{-\theta\left(T_{I}^{*}(\underline{x})\right)} .
\end{aligned}
$$

The Bayes estimator $\hat{\hat{\theta}}_{I C}$ for unknown parameter $\theta$ under LLF is obtain by simplifying following equality

$$
\begin{aligned}
& \hat{\hat{\theta}}_{I C}=-\frac{1}{a} \log E\left(e^{-a \theta}\right) \\
& \Rightarrow \hat{\hat{\theta}}_{I C}=\left(\frac{r}{a}\right) \log \left(1+\frac{a}{T_{I}^{*}(\underline{x})}\right) .
\end{aligned}
$$

\subsection{Doubly Type-II Censoring}

Using joint probability density function (2.6) and equation (1.3) in equation (2.2), posterior density and Bayes estimator $\hat{\theta}_{D C}$ corresponding to unknown parameter $\theta$ under LLF are given as 


$$
\begin{array}{r}
\pi_{D C}^{* *}(\theta \mid \underline{x})=\hat{\eta} \sum_{k=0}^{r-1}(-1)^{k}{ }^{r-1} C_{k} \theta^{s-r} \exp \left(-\theta\left(T_{D}^{*}(\underline{x})\right)\right) ; \\
\hat{\eta}=\left(\sum_{k=0}^{r-1}(-1)^{k}{ }^{r-1} C_{k} \frac{\Gamma(s-r+1)}{\left(T_{D}^{*}(\underline{x})\right)^{(s-r+1)}}\right)^{-1} \text { and } \\
\hat{\hat{\theta}}_{D C}=-\frac{1}{a} \log \left(\hat{\eta}^{*} \sum_{k=0}^{r-1}(-1)^{k}{ }^{r-1} C_{k}\left(T_{D}^{*}(\underline{x})+a\right)^{-(s-r+1)}\right)
\end{array}
$$

where $\hat{\eta}^{*}=\left(\sum_{k=0}^{r-1}(-1)^{k}{ }^{r-1} C_{k}\left(T_{D}^{*}(\underline{x})\right)^{-(s-r+1)}\right)^{-1}$.

\subsection{Progressive Type-II Censoring}

On similar line, the posterior density corresponding to prior (1.3) under Progressive censoring is obtained as

$$
\Rightarrow \pi_{P C}^{* *}(\theta \mid \underline{x})=\frac{\left(T_{P}^{*}(\underline{x})\right)^{r}}{\Gamma(r)} \theta^{r-1} e^{-\theta\left(T_{P}^{*}(\underline{x})\right)}
$$

and the Bayes estimator $\hat{\theta}_{P C}$ under LLF for unknown parameter $\theta$ is

$$
\Rightarrow \hat{\hat{\theta}}_{P C}=\left(\frac{r}{a}\right) \log \left(1+\frac{a}{T_{P}^{*}(\underline{x})}\right) .
$$

\section{Simulation Study}

A simulation is used in order to compare the performances of the proposed Bayes estimators in terms of risk magnitude under different censored plans. We carry out this comparison by taking the sample size as $n=20$ with the set of prior parameter $(b, c)=(0.50,0.70),(1.00,1.00)$, $(2.50,1.58),(5,2.30),(10,3.16)$ and $(0,0)$. Here, the criterion behind the selection of these prior parametric values is that the prior variance should be unity. Also, $b=c=0$ reflect the study under non-informative (Jeffrey's) prior. Hence, all the results should be valid for both informative and non-informative priors.

Tables 1-3, presents the risk of Bayes estimators obtained under LLF using Item-Failure, Doubly and Progressive Type-II censoring scheme respectively for selected set of values of $a$ $(=0.50,1.00,1.50)$ and $\theta(=1.00,2.50,500)$. The results are based on 100,000 simulation runs. 
The Gompertz distribution is a flexible distribution that can be skewed to the right and to the left. The Gompertz distribution is often applied to describe the distribution of adult lifespans by actuaries and demographers. Computer scientists have also started to model the failure rates of computer codes by the Gompertz distribution. Related fields of science such as biology and gerontology also considered the Gompertz distribution for the analysis of survival. More recently, In Marketing Science, it has been used as an individual-level simulation for customer lifetime value modeling. All the said applications, overestimation is more serious than underestimation.

Hence, the positive value of shape parameter $a(>0)$ is considered here and it shows the loss function is quite asymmetric about 0 with overestimation being more costly than underestimation.

\subsection{Item-Failure Censoring Scheme}

The test is terminated when $r(=5,10,15)$, as it is supposed. The risk magnitude is increase as prior parameter increase (except for $(b, c)=(10,3.16)$ ). A decreasing trend has seen in the risk magnitude when censored sample size $r$ increases when other parametric values are fixed (Table 1). Further an opposite trend in the risk magnitude also seen when ' $a$ ' increase.

It is noted that, when the value of parameter $\theta$ increases the risk magnitude first decrease and then increase. However, the magnitude of risk under the LLF is nominal (Table 1).

\subsection{Doubly Censoring Scheme}

The Risk of the Bayes estimators $\hat{\theta}_{D C}$ and $\hat{\hat{\theta}}_{D C}$ have been obtained under LLF, for the similar set of considered parametric values as discussed above and presented in Table 2 . The left censoring rate is assumed to be $20 \%, 30 \%, 40 \%$; and the right censoring rate accordingly assume for the fixed non-censored items 5, 10, 15 respectively. For example if the left censoring rate is $20 \%$ and the non-censored items is prefixed into 5, then right censoring rate will be $55 \%$.

All the behaviors have been seen similar corresponding to the Bayes estimators obtained under Item-Failure criterion. The gain in risk magnitude is noted here for doubly censoring criterion as compared to previous one. However, the gain in magnitude is robust.

\subsection{Progressive Censoring Scheme}

A Progressively Type-II censored sample of size $r$ form given values of censoring scheme $R_{i} ; i=1,2, \ldots, r$, for considered model have generated, according to an algorithm proposed by Balakrishnan \& Aggarwala (2000). The censoring schemes for different values of $r$ are presented in Table 7 and the risk of Bayes estimators under LLF in Table 3. 
Again it is seen that all the properties of Bayes estimators are similar as discussed above, for both censoring criterion. Further, it is noted also here that the magnitude of risk are smaller than compared to Item Failure or doubly censoring criterion.

\subsection{Studies with Generated Values of}

For observing the effect of the unknown parameter $\theta$, we generate the value of parameter $\theta$ by using $b$ and $c$ from prior density (1.2). Using these generated values of $\theta$ and other parametric values considered as above, the risk of Bayes estimators obtained and presented in Tables 4-6 under all three considered censoring criterion. All the properties have been seen similar as discussed above. It is also noted that the risk magnitude of Bayes estimators is robust when compared to pre-assume values of parameter $\theta$ case, for all considered censoring plans.

\section{A Real Data Example}

We illustrate the use of estimation methods given in this paper by real data considered by King et al. (1979) for a tumor-free days of 30 rats fed with unsaturated diet. These data are also presented by Lee (1992) and studied by Chen (1997) and Wu et al. (2003). They assumed a Gompertz distribution for tumor-free times.

Under the different censoring plans, the censored sample size $r$ is taken as 18 from $n=30$. The risks magnitude of Bayes estimators under LLF are presented in Table 8, with others parametric values as considered in previous section. All the properties as discussed above have been seen similar. Also, the risks magnitude obtained here are least than compared to simulation study. This pattern is similar under each censoring plans.

\section{Conclusion}

The censoring arises when exact lifetimes are only partially known and it is useful in life testing experiments for time and cost restrictions. Especially when some sample values at either or both extremes might have been adulterated. The aim of article is to present a comparative study based on Bayes estimation under different censoring plans. For this we consider here Item failure censoring, Doubly and Progressive Type-II right censoring. The Bayes estimation has been addressed for underlying model under the LINEX loss function.

Here, two different criterions for selection of values of unknown parameter has been taken, in which one is pre assumed value of unknown parameter and second one is the generated values of the unknown parameter. From the above sections we have already seen that all properties of Bayes estimators under all censoring plans are seen to be similar. But, one remarkable point is that the risk magnitude of Bayes estimators under LLF is nominal when it is taken from 
Progressive Type-II censoring. In comparison of censoring schemes, the result under Progressive Type-II censoring seems to be more efficient over Item-failure or Doubly censoring scheme.

It has been seen also that, when the shape parameter of LINEX loss ' $a$ ' increase the risk first increase and then stable. For higher values of ' $a$ ', the changes in magnitude of the risk is robust. Hence, small values of shape parameter are suitable.

\section{Acknowledgement}

Author is highly thankful to the editor and referees for their valuable suggestions without which the paper could not have taken its present form.

\section{References}

[1] Ananda, M. M., Dalpatadu, R. J. \& Singh, A. K. (1996). Adaptive Bayes estimators for parameters of the Gompertz survival model. Applied Mathematics and Computation, 75(2), $167-177$.

[2] Balakrishnan, N. \& Aggarwala, R. (2000). Progressive Censoring: Theory, Methods and Applications. Birkhauser Publishers, Boston.

[3] Chen. Z. (1997). Parameter estimation of the Gompertz population. Biometrical Journal, 39 (1), 117-124.

[4] Gompertz, B. (1825). On the nature of the function expressive of the Law of Human Mortality, and on a new mode of determining the value of life contingencies. Philosopher Transaction Royal Society of London, 115, 513-585.

[5] Gordon, N. H. (1990). Maximum likelihood estimation for mixtures of two Gompertz distribution when censoring occurs. Communication in Statistics - Simulation and Computation, 19, 733-747.

[6] Ismail, A. A. (2010). Bayes estimation of Gompertz distribution parameters and acceleration factor under partially accelerated life tests with type-I censoring. Journal of Statistical Computation and Simulation, 80 (11), 1253-1264.

[7] King, M. M., Bailey, D. M., Gibson, D. D., Pitha, J. V. \& McCay, P. B. (1979). Incidence and growth of mammary tumors induced by 7, 12-dimethylbenz ${ }^{[\alpha]}$ anthracene as related to the dietary content of fat and antioxidant. Journal of the National Cancer Institute, $\mathbf{6 3}$ (3), 657-663. 
[8] Jaheen Z. F. (2003). A Bayesian analysis of record statistics from the Gompertz model. Applied Mathematics and Computation, 145(2), 307-320.

[9] Lee, E. T. (1992). Statistical methods for survival data analysis-2nd edition. Wiley, New York.

[10] Soliman, A. A., Abd-Ellah, A. H., Abou-Elheggag, N. A. \& Abd-Elmougod, G. A. (2012). Estimation of the parameters of life for Gompertz distribution using progressive first-failure censored data. Computational Statistical Data Analysis, 56(8), 2471-2485.

[11]Wu, S. J., Chang, C. T. \& Tsai, T. R. (2003). Point and interval estimations for the Gompertz distribution under progressive type-II censoring. Metron, 61, 403-418.

Received January 20, 2015; accepted March 24, 2015.

Gyan Prakash

Department of Community Medicine

S. N. Medical College, Agra, U. P., India 
Table 1: Risk of Bayes Estimator under Item-Failure Censoring

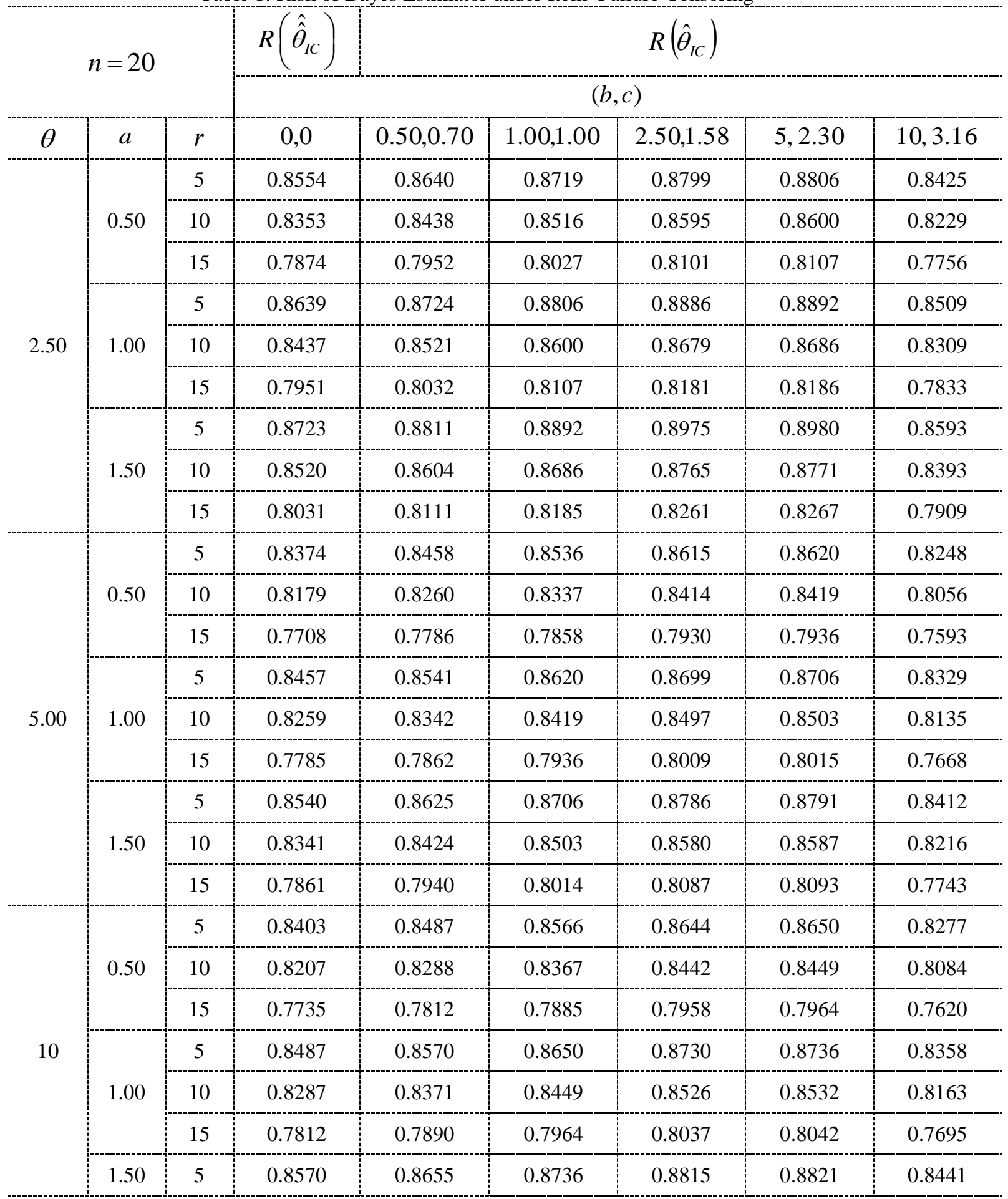


274 A COMPARATIVE STUDY BASED ON BAYES ESTIMATION UNDER DIFFERENT CENSORING CRITERION

\begin{tabular}{|l|c|c|c|c|c|c|c} 
& 10 & 0.8371 & 0.8453 & 0.8532 & 0.8611 & 0.8618 & 0.8244 \\
\hline & 15 & 0.7889 & 0.7968 & 0.8041 & 0.8115 & 0.8122 & 0.7770 \\
\hline
\end{tabular}


Table 2: Risk of Bayes Estimator under Doubly Censoring

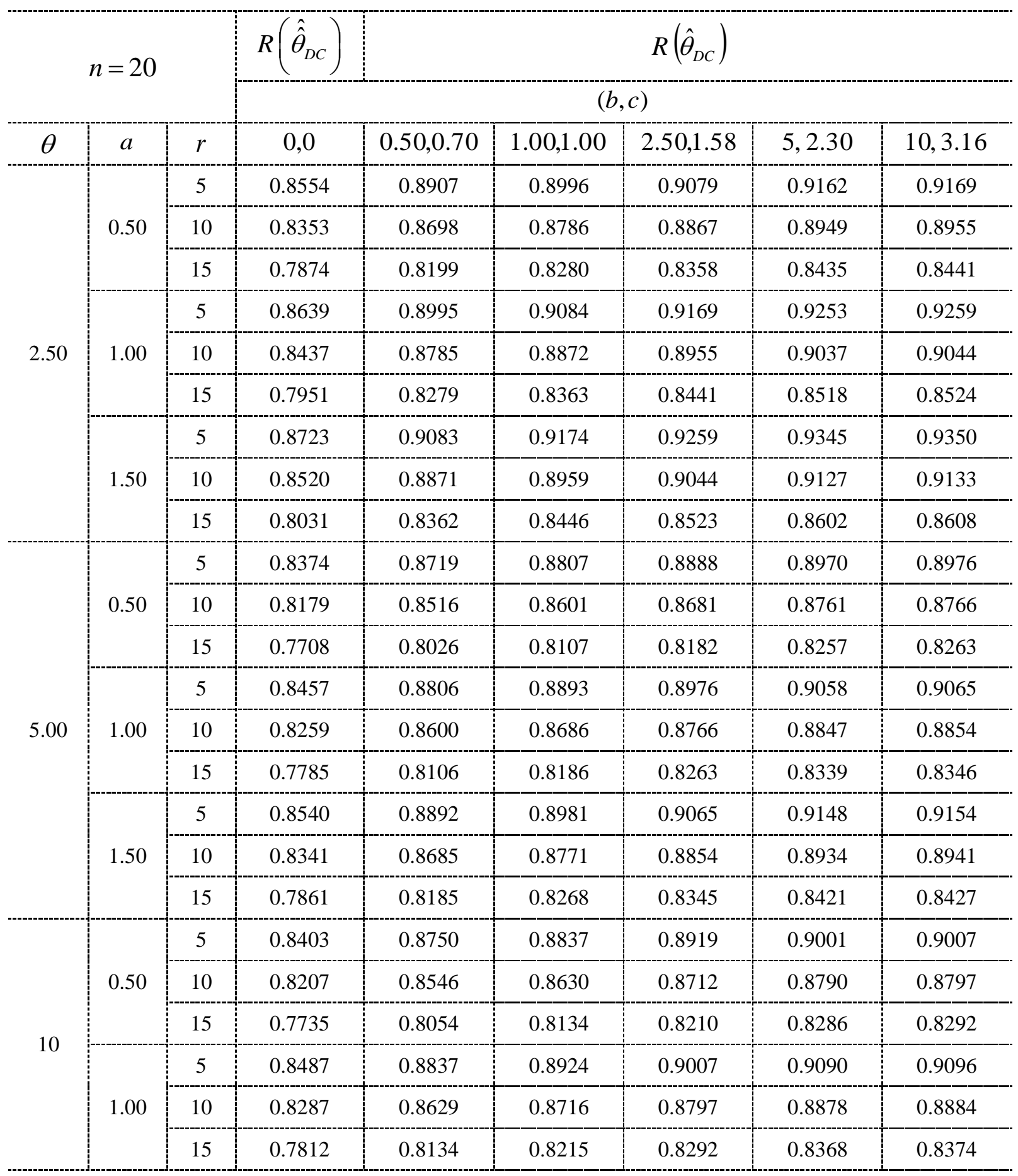


276 A COMPARATIVE STUDY BASED ON BAYES ESTIMATION UNDER DIFFERENT CENSORING CRITERION

\begin{tabular}{|c|c|c|c|c|c|c|c}
\hline \multirow{2}{*}{1.50} & 5 & 0.8570 & 0.8923 & 0.9012 & 0.9096 & 0.9179 & 0.9185 \\
\hline & 10 & 0.8371 & 0.8716 & 0.8802 & 0.8884 & 0.8966 & 0.8973 \\
\hline & 15 & 0.7889 & 0.8214 & 0.8297 & 0.8373 & 0.8450 & 0.8457 \\
\hline
\end{tabular}




\begin{tabular}{|c|c|c|c|c|c|c|c|c|}
\hline \multirow{2}{*}{\multicolumn{3}{|c|}{$n=20$}} & $R\left(\hat{\hat{\theta}}_{P C}\right)$ & \multicolumn{5}{|c|}{$R\left(\hat{\theta}_{P C}\right)$} \\
\hline & & & \multicolumn{6}{|c|}{$(b, c)$} \\
\hline$\theta$ & $a$ & $r$ & 0,0 & $0.50,0.70$ & $\begin{array}{c}1.00,1.00 \\
\text { kash }\end{array}$ & $2.50,1.58$ & $5,2.30$ & $10,3.16$ \\
\hline \multirow{9}{*}{2.50} & \multirow{3}{*}{0.50} & 5 & 0.7890 & 0.7969 & 0.8042 & 0.8115 & 0.8122 & 0.7770 \\
\hline & & 10 & 0.7704 & 0.7783 & 0.7855 & 0.7928 & 0.7932 & 0.7590 \\
\hline & & 15 & 0.7262 & 0.7334 & 0.7404 & 0.7472 & 0.7478 & 0.7154 \\
\hline & \multirow{3}{*}{1.00} & 5 & 0.7968 & 0.8046 & 0.8122 & 0.8196 & 0.8202 & 0.7848 \\
\hline & & 10 & 0.7782 & 0.7859 & 0.7932 & 0.8005 & 0.8012 & 0.7664 \\
\hline & & 15 & 0.7334 & 0.7408 & 0.7478 & 0.7546 & 0.7551 & 0.7225 \\
\hline & \multirow{3}{*}{1.50} & 5 & 0.8045 & 0.8127 & 0.8202 & 0.8278 & 0.8282 & 0.7926 \\
\hline & & 10 & 0.7858 & 0.7936 & 0.8012 & 0.8085 & 0.8090 & 0.7742 \\
\hline & & 15 & 0.7407 & 0.7481 & 0.7550 & 0.7620 & 0.7625 & 0.7295 \\
\hline \multirow{9}{*}{5.00} & \multirow{3}{*}{0.50} & 5 & 0.7723 & 0.7801 & 0.7873 & 0.7946 & 0.7951 & 0.7607 \\
\hline & & 10 & 0.7544 & 0.7619 & 0.7690 & 0.7761 & 0.7765 & 0.7431 \\
\hline & & 15 & 0.7110 & 0.7182 & 0.7248 & 0.7314 & 0.7320 & 0.7003 \\
\hline & \multirow{3}{*}{1.00} & 5 & 0.7800 & 0.7878 & 0.7951 & 0.8023 & 0.8030 & 0.7682 \\
\hline & & 10 & 0.7618 & 0.7695 & 0.7765 & 0.7837 & 0.7843 & 0.7504 \\
\hline & & 15 & 0.7181 & 0.7252 & 0.7320 & 0.7387 & 0.7393 & 0.7072 \\
\hline & \multirow{3}{*}{1.50} & 5 & 0.7877 & 0.7955 & 0.8030 & 0.8104 & 0.8109 & 0.7759 \\
\hline & & 10 & 0.7694 & 0.7770 & 0.7843 & 0.7914 & 0.7920 & 0.7578 \\
\hline & & 15 & 0.7251 & 0.7323 & 0.7392 & 0.7459 & 0.7464 & 0.7141 \\
\hline \multirow{9}{*}{10} & \multirow{3}{*}{0.50} & 5 & 0.7750 & 0.7828 & 0.7901 & 0.7973 & 0.7978 & 0.7634 \\
\hline & & 10 & 0.7570 & 0.7645 & 0.7718 & 0.7787 & 0.7793 & 0.7456 \\
\hline & & 15 & 0.7135 & 0.7206 & 0.7273 & 0.7340 & 0.7346 & 0.7028 \\
\hline & \multirow{3}{*}{1.00} & 5 & 0.7828 & 0.7905 & 0.7978 & 0.8052 & 0.8058 & 0.7709 \\
\hline & & 10 & 0.7644 & 0.7721 & 0.7793 & 0.7864 & 0.7869 & 0.7529 \\
\hline & & 15 & 0.7206 & 0.7277 & 0.7346 & 0.7413 & 0.7417 & 0.7097 \\
\hline & \multirow{3}{*}{1.50} & 5 & 0.7905 & 0.7983 & 0.8058 & 0.8131 & 0.8136 & 0.7786 \\
\hline & & 10 & 0.7721 & 0.7796 & 0.7869 & 0.7942 & 0.7949 & 0.7603 \\
\hline & & 15 & 0.7276 & 0.7349 & 0.7416 & 0.7485 & 0.7491 & 0.7166 \\
\hline
\end{tabular}

Table 3: Risk of Bayes Estimator under Progressive Censoring 
Table 4: Risk of Bayes Estimator Based on Generated $\theta$ (Item-Failure Censoring)

\begin{tabular}{|c|c|c|c|c|c|c|c|}
\hline \multicolumn{2}{|c|}{$n=20$} & $R\left(\hat{\hat{\theta}}_{I C}\right)$ & \multicolumn{5}{|c|}{$R\left(\hat{\theta}_{I C}\right)$} \\
\hline & & \multicolumn{6}{|c|}{$(b, c)$} \\
\hline$a$ & $r$ & 0,0 & $0.50,0.70$ & $1.00,1.00$ & $2.50,1.58$ & $5,2.30$ & $10,3.16$ \\
\hline \multirow{3}{*}{0.50} & 5 & 0.7846 & 0.7925 & 0.7999 & 0.8073 & 0.8078 & 0.7728 \\
\hline & 10 & 0.7664 & 0.7740 & 0.7812 & 0.7884 & 0.7888 & 0.7549 \\
\hline & 15 & 0.7223 & 0.7296 & 0.7363 & 0.7431 & 0.7437 & 0.7115 \\
\hline \multirow{3}{*}{1.00} & 5 & 0.7924 & 0.8003 & 0.8078 & 0.8151 & 0.8158 & 0.7804 \\
\hline & 10 & 0.7739 & 0.7817 & 0.7888 & 0.7962 & 0.7967 & 0.7623 \\
\hline & 15 & 0.7295 & 0.7367 & 0.7437 & 0.7505 & 0.7511 & 0.7185 \\
\hline \multirow{3}{*}{1.50} & 5 & 0.8002 & 0.8082 & 0.8158 & 0.8233 & 0.8238 & 0.7882 \\
\hline & 10 & 0.7816 & 0.7893 & 0.7967 & 0.8040 & 0.8046 & 0.7699 \\
\hline & 15 & 0.7366 & 0.7440 & 0.7510 & 0.7578 & 0.7583 & 0.7255 \\
\hline
\end{tabular}

Table 5: Risk of Bayes Estimator Based on Generated $\theta$ (Doubly Censoring)

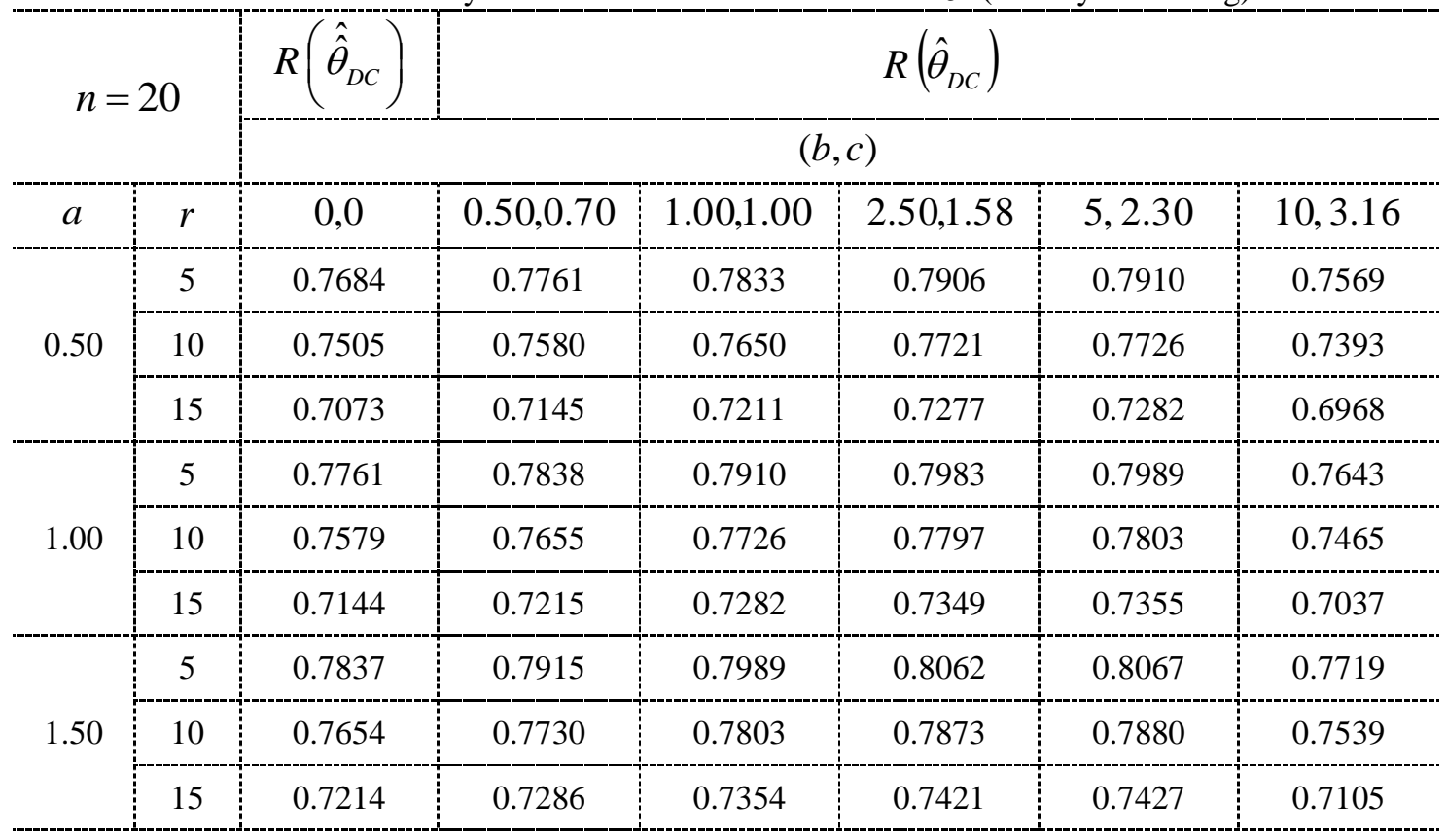


Table 6: Risk of Bayes Estimator Based on Generated $\theta$ (Progressive Censoring)

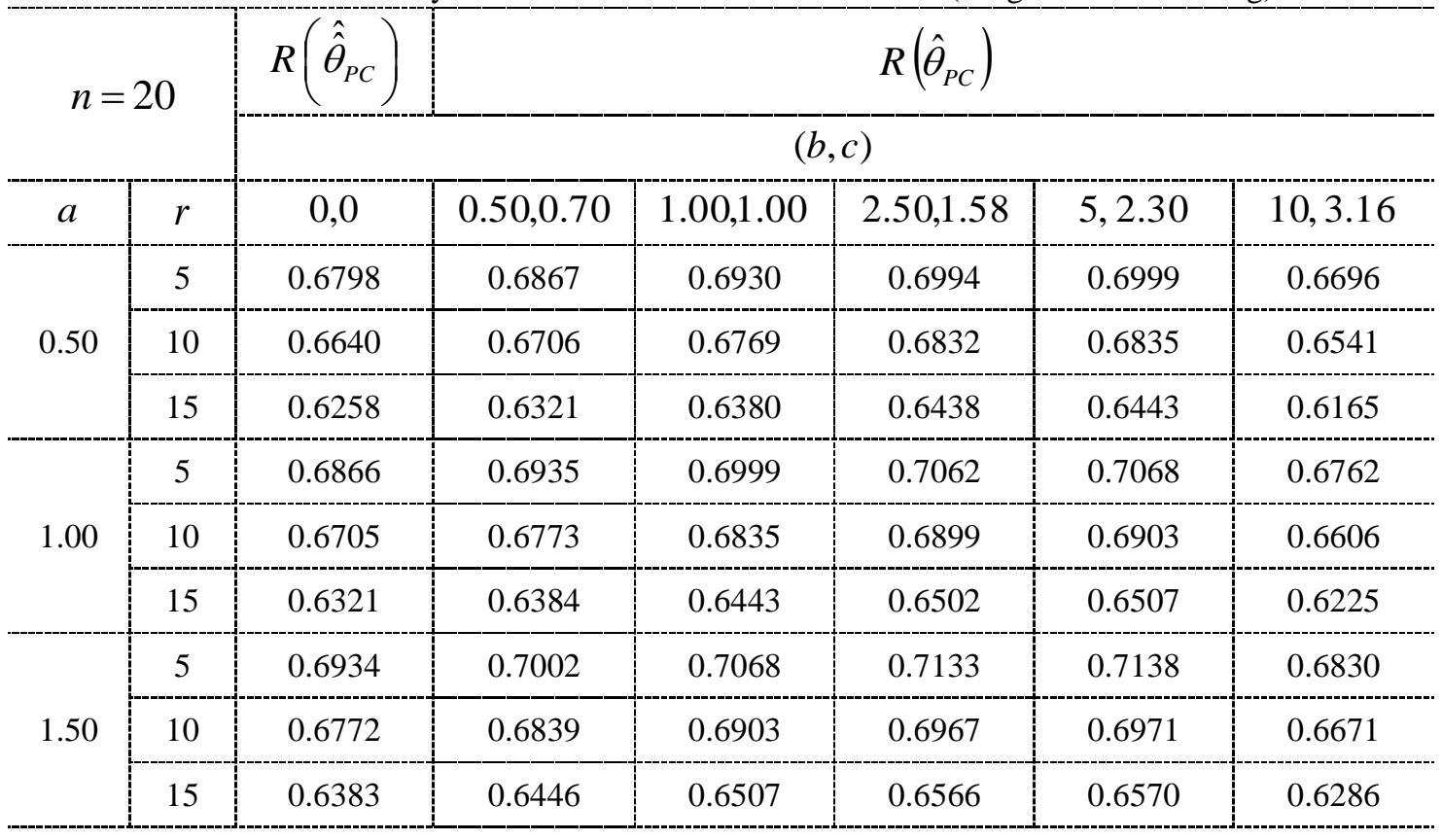

Table 7: Different Progressive Censoring Scheme

\begin{tabular}{c|c|c}
\hline Case & $\mathrm{r}$ & $\mathrm{R}_{\mathrm{i}} ; \mathrm{i}=1,2, \ldots, \mathrm{r}$ \\
\hline 1 & 5 & 12101 \\
\hline 2 & 10 & 1003001001 \\
\hline 3 & 15 & 102001020001001 \\
\hline
\end{tabular}


Table 8: The Risk of Bayes Estimators Based on Real Data

\begin{tabular}{|c|c|c|c|c|c|c|}
\hline \multicolumn{7}{|c|}{ Item-Failure Censoring } \\
\hline \multirow{2}{*}{$\begin{array}{l}n=30 \\
r=18\end{array}$} & $R\left(\hat{\hat{\theta}}_{I C}\right)$ & \multicolumn{5}{|c|}{$R\left(\hat{\theta}_{I C}\right)$} \\
\hline & \multicolumn{6}{|c|}{$(b, c)$} \\
\hline$a$ & 0,0 & $0.50,0.70$ & $1.00,1.00$ & $2.50,1.58$ & $5,2.30$ & $10,3.16$ \\
\hline 0.50 & 0.7469 & 0.7544 & 0.7614 & 0.7685 & 0.7690 & 0.7357 \\
\hline 1.00 & 0.7543 & 0.7618 & 0.7690 & 0.7760 & 0.7766 & 0.7429 \\
\hline 1.50 & 0.7617 & 0.7693 & 0.7766 & 0.7838 & 0.7842 & 0.7503 \\
\hline \multicolumn{7}{|c|}{ Doubly Censoring } \\
\hline \multirow{2}{*}{$\begin{array}{l}n=30 \\
r=18\end{array}$} & $R\left(\hat{\hat{\theta}}_{D C}\right)$ & \multicolumn{5}{|c|}{$R\left(\hat{\theta}_{D C}\right)$} \\
\hline & \multicolumn{6}{|c|}{$(b, c)$} \\
\hline$a$ & 0,0 & $0.50,0.70$ & $1.00,1.00$ & $2.50,1.58$ & $5,2.30$ & $10,3.16$ \\
\hline 0.50 & 0.7137 & 0.7208 & 0.7275 & 0.7343 & 0.7347 & 0.7030 \\
\hline 1.00 & 0.7208 & 0.7280 & 0.7347 & 0.7415 & 0.7420 & 0.7099 \\
\hline 1.50 & 0.7279 & 0.7351 & 0.7420 & 0.7488 & 0.7493 & 0.7169 \\
\hline \multicolumn{7}{|c|}{ Progressive Censoring } \\
\hline \multirow{2}{*}{$\begin{array}{l}n=30 \\
r=18\end{array}$} & $R\left(\hat{\hat{\theta}}_{P C}\right)$ & \multicolumn{5}{|c|}{$R\left(\hat{\theta}_{P C}\right)$} \\
\hline & \multicolumn{6}{|c|}{$(b, c)$} \\
\hline$a$ & 0,0 & $0.50,0.70$ & $1.00,1.00$ & $2.50,1.58$ & $5,2.30$ & $10,3.16$ \\
\hline 0.50 & 0.6472 & 0.6537 & 0.6597 & 0.6658 & 0.6663 & 0.6374 \\
\hline 1.00 & 0.6536 & 0.6601 & 0.6663 & 0.6722 & 0.6728 & 0.6438 \\
\hline 1.50 & 0.6600 & 0.6666 & 0.6728 & 0.6790 & 0.6795 & 0.6502 \\
\hline
\end{tabular}

